\title{
Organic Synthesis via Irradiation and Warming of Ice Grains in the Solar Nebula
}

\author{
Fred J. Ciesla, ${ }^{1 *}$ Scott A. Sandford ${ }^{2}$ \\ ${ }^{1}$ Department of the Geophysical Sciences, University of Chicago, \\ 5734 South Ellis Avenue, IL 60430, USA \\ ${ }^{2}$ NASA Ames Research Center, MS 245-6, Moffett Field, CA 94035, USA
}

*To whom correspondence should be addressed; E-mail: fciesla@uchicago.edu

Complex organic compounds, including many important to life on Earth, are commonly found in meteoritic and cometary samples, though their origins remain a mystery. We examined whether such molecules could be produced within the solar nebula by tracking the dynamical evolution of ice grains in the nebula and recording the environments they were exposed to. We found that icy grains originating in the outer disk, where temperatures were $<30$ $K$, experienced UV irradiation exposures and thermal warming similar to that which has been shown to produce complex organics in laboratory experiments. These results imply that organic compounds are natural byproducts of protoplanetary disk evolution and should be important ingredients in the formation of all planetary systems, including our own.

Complex organic compounds have been found in meteorites and interplanetary dust particles, leading many to suggest that Earth's prebiotic material was delivered by comets and chondritic-like planetesimals. The origin of these organic compounds, however, is unknown. 
While organic molecules can be produced during aqueous alteration of a parent body (1), or through Fischer-Tropsch reactions in protoplanetary disks (2), the high $\mathrm{D} / \mathrm{H}$ ratios found in meteoritic organics points to a low temperature origin (3). Irradiation of astrophysical ice analogues (containing simple molecules like $\mathrm{H}_{2} \mathrm{O}, \mathrm{CO}, \mathrm{CO}_{2}, \mathrm{NH}_{3}$ and $\mathrm{CH}_{3} \mathrm{OH}$ ) by $\mathrm{UV}$ photons in the laboratory have produced of a suite of organic molecules similar to those found in primitive bodies in our Solar System, including amino acids, amphiphiles, quinones, and nucleobases (410). These experiments simulated conditions (temperatures, pressures, radiation fluxes) similar to those found in dark molecular clouds-the collection of gas and dust that collapse to form stars and planets. Within these clouds, ice-mantled grains are exposed to relatively low fluxes of UV from the ambient interstellar radiation field, allowing organics to be produced. It remains unclear, however, if the full suite of complex organics found in primitive bodies could be produced within a molecular cloud, and to what extent they were preserved during the formation of the Solar System.

These experimental conditions are also relevant to the outer solar nebula, where temperatures were low enough for $\mathrm{C}-, \mathrm{H}-, \mathrm{O}-$, and $\mathrm{N}$-bearing ices to form (11). If ices in this region were irradiated to substantial levels, organics would have been produced, increasing the inventory of such compounds beyond what may have been inherited from the molecular cloud. Indeed, observations of molecular emission features suggest that protoplanetary disks support active organic chemistry, processing the raw materials from the molecular cloud to yield higher abundances of organic species, though the pathways for production are unclear (12).

To investigate the production of organics after molecular cloud collapse, we modeled the paths of ice-mantled grains through the solar nebula (13-16). Particles migrated around the nebula due to disk evolution, gas drag, gravitational settling, and both radial and vertical turbulent diffusion. We assumed that the level of turbulence in the disk is characterized by the turbulence parameter, $\alpha$, which describes both the viscosity in the disk, $\nu=\alpha c_{s} H$ where $c_{s}$ is the 
local speed of sound in the disk and $H$ is the local gas scale height, and the diffusivity of trace materials, $D(\sim \nu)(16,17)$. We set $\alpha=0.001$ everywhere, as the magnetorotational instability is expected to have operated in the outer disk (18).

The disk was assumed to be in steady-state and had a surface density distribution $\Sigma(r)=$ $2000\left(\frac{r}{1 \mathrm{AU}}\right)^{-1} \mathrm{~g} / \mathrm{cm}^{2}$ (amounting to $0.1 M_{\odot}$ out to $70 \mathrm{AU}$ ) and a thermal structure $T(r)=$ $200\left(\frac{r}{1 \mathrm{AU}}\right)^{-\frac{1}{2}} \mathrm{~K}$, appropriate for flared disks around young T-Tauri stars $(11,19)$. The dynamics of 5000 ice-mantled grains with radius $a=1 \mu \mathrm{m}$ and unit density were tracked. While particles of a broad range of sizes would have been present, we chose to follow $1 \mu \mathrm{m}$ particles as individual organic hot spots and globules in meteorites and comets are often found to be of this spatial scale $(20,21)$. Particles began at the midplane of the disk ( $z=0 \mathrm{AU})$ where $T=30 \mathrm{~K}(r=49 \mathrm{AU})$. At this temperature, $\mathrm{C}$ - and $\mathrm{N}$ - bearing ices of the type needed to form organics condensed (22) and $\mathrm{D} / \mathrm{H}$ ratios in water are elevated due to ion-molecule reactions (23).

As a particle moved through the disk, we calculated the number of UV photons incident on its surface. The radiation was assumed to be incident on the disk with a flux, $F_{0}$, directed normal to the disk midplane. The flux was attenuated with depth as photons were absorbed by solid particles. Thus, the flux everywhere was $F(r, z)=F_{0} e^{-\tau(r, z)}$, where $\tau$ was the optical depth of material suspended in the disk above the point of interest $\left(\tau(r, z)=\int_{|z|}^{\infty} \rho_{g}(r, z) \kappa d z\right.$, where $\rho_{g}(r, z)$ is the density of material in the disk and $\kappa$ is the average opacity of the suspension). We assume an opacity of $\kappa=7.5 \mathrm{~cm}^{2} / \mathrm{g}$, a conservative value which is on the high end of the range expected in the cool outer regions of a protoplanetary disk (24). Lower opacities would increase the number of photons seen by each particle. We take as a baseline an incident flux equivalent to the current interstellar UV flux of $10^{8}$ photons $\mathrm{cm}^{-2} \mathrm{~s}^{-1}\left(1 G_{0}\right)(25)$, but higher fluxes can be considered by multiplying by the desired enhancement factor.

The starting location of the particles was shielded such that the flux was attenuated by over 25 orders of magnitude. As most of the mass of the nebula was located around the midplane, 
these conditions are often used in calculating the chemical evolution of primitive materials. At this location, an ice grain would have seen $<10^{-25} F_{0} \pi a^{2} \Delta t \sim 10^{-11}$ photons if it remained at its starting location throughout the simulation, a period of $\Delta t=10^{6}$ years. However, in the presence of vertical diffusion small particles spend $\sim 68 \%$ of their time within $1 H$ of the midplane, but $32 \%$ of their lifetimes at higher altitudes, with $\sim 5 \%$ being spent above $2 H(14)$. Because the UV flux was a strong function of height, it was the infrequent, but non-negligible, excursions to high altitudes that were primarily responsible for small grains seeing large numbers of photons. Irradiation exposures also increased as grains diffused outward in the disk, where the lower surface densities of gas and dust allowed the UV to penetrate closer to the disk midplane. Thus the radial and vertical motions of the grains controlled their UV exposures.

Figure 2 shows that the cumulative number of UV photons grains encountered did not climb uniformly. Rather, the increases in incident photons were episodic and occurred when a grain was lofted to high altitudes or far out in the disk. Plotting the irradiation histories against the temperatures seen by the particles, where we took the particle temperature as being equal to the gas temperature everywhere, demonstrates how the UV exposure generally rose early while the grains were cold and when all key ices remained on the grain. Less than $0.5 \%$ of photons would desorb these molecules (26) which would then recondense on the same or another grain. Thus loss of mass in this manner would be minimal.

Figure 3 shows that a typical dosage for the particles was $\sim 5 \times 10^{12}$ photons in our nominal case. Assuming a pure $\mathrm{H}_{2} \mathrm{O}$ grain, this corresponds to $\sim 50 \mathrm{UV}$ photons/molecule. Experimental results suggest an efficiency of production of $\sim 1$ organic molecule produced per 400 incident photons $(6,16)$. Thus $>12 \%$ of the mass of ices in the outer solar nebula would be converted to organic compounds. However, protoplanetary disks like our solar nebula are exposed to enhanced UV fluxes, either from their own central stars (27), or from other stars in even a low mass cluster, resulting in an average incident fluxes of up to $\sim 10^{3} G_{0}(28)$. This increases the 
average dosage of ice grains to 50,000 photons/molecule, greatly increasing organic production.

Grain growth would have resulted in lower photon dosages due to decreased particle crosssections per unit volume $(16,29)$. However, the decrease in opacity of the nebula would have allowed UV photons to penetrate deeper into the disk (29) to irradiate the grain aggregates. Further, models of dust growth show that fragmentation would constantly free particles from the larger aggregates through energetic collisions (30). Thus, even if icy grains remain as $1 \mu \mathrm{m}$ entities for just a part of the $10^{6}$ years modeled here, they can receive substantial UV dosages (Fig. 2).

Irradiation, by itself, does not produce the organic compounds. When UV photons are incident on astrophysical ices in the laboratory, they break molecular bonds in the ices, producing reactive ions and radicals. If these reactive photoproducts are adjacent to each other, they may react even at very low temperatures, forming new compounds. When the irradiated ice is warmed, the photoproducts become mobile and photoproducts react more readily. Such reactions occur during warm-ups through any temperature range, but are particularly enhanced during the amorphous-amorphous transition near $80 \mathrm{~K}$, the amorphous-to-cubic transformation around $135 \mathrm{~K}$, and the sublimation of the $\mathrm{H}_{2} \mathrm{O}$ in the ice at $\mathrm{T}>135 \mathrm{~K}(4-10,16)$. Thus, warming of irradiated ices yields a burst of organic synthesis, even if the ice is not currently being irradiated. Reactions brought on by warming are still largely constrained by proximity, rather than considerations of normal chemical equilibrium. However, these experimental results are robust in that the amounts and kinds of new molecules produced is largely insensitive to the temperature of the ices during irradiation and the exact composition of the starting ices (16).

Particle warming would have occurred as grains diffused to high altitudes where the increased photon flux would have heated grains beyond the midplane temperature. While we assumed our disk was isothermal with height, temperatures may reach $\sim 10-50 \mathrm{~K}$ above midplane temperatures at heights of $|z|>0.2 r$ outside $\sim 10$ AU (31). As such, not only would 
particles accumulate substantial UV dosages, but they would also be warmed as they moved through the disk. Thus, the conditions shown to produce organics in laboratory experiments, irradiation and warming, would be natural consequences of the dynamical evolution of ices in the solar nebula. Photo-processing in the solar nebula thus represents an ideal environment for this form of organic synthesis.

That the grains in our study are irradiated during their large-scale movement in the disk suggests that similar products would be made available to meteoritic and cometary bodies, consistent with observations $(20,21)$. Further, organics on particles brought to the innermost regions of a protoplanetary disk would vaporize, allowing the products of this organic chemistry to be observed in the gas phase, offering an explanation for the emission features observed by Spitzer (12). As we predict that complex species such as amino acids will be formed, identification of more complex organic species in other disks may serve to test this method of production. Because the total UV fluences ices received in the solar nebula are considerably greater than experienced by typical ices in dense molecular clouds, disk processing would yield greater overall conversions of ices into organics, and produce organics of greater complexity. The exact mass of organic production remains uncertain, as it will depend on the incident UV flux and the mass of ice contained in the outer disk. Our results, however, demonstrate that cold, icy $1 \mu \mathrm{m}$ grains would see UV dosages of $\sim 10^{12}-10^{15}$ photons for modest incident fluxes of $1-10^{2} G_{0}$. This allows for substantial organic production, with at least $\sim 5 \%$ of the mass of ices being converted to organics and production being limited more by the availability of starting materials than energetic photons (16). These fluxes are much less than it was thought to be necessary for organic production to occur in protoplanetary disks $\left(\sim 10^{6} G_{0} ; 29\right)$. Thus, rather than being limited to disks around stars located near massive $\mathrm{O} / \mathrm{B}$ stars, complex organics should be produced in disks around all stars and made available for delivery to the surface of planets where they could play a role in the origin of life. 


\section{References and Notes}

1. E.T. Peltzer et al. Advances in Space Research 4, 69 (1984).

2. J.A. Nuth, N.M. Johnson, S. Manning Astrophysical Journal 673, L225 (2008).

3. S.A. Sandford, M.P. Bernstein, J.P. Dworkin, Meteoritics and Planetary Science 36, 1117 (2001).

4. W. Hagen, L.J. Allamandola, J.M. Greenberg, Astrophysics and Space Science 65, 215 (1979).

5. W.A. Schutte, L.J. Allamandola, S.A. Sandford, Advances in Space Research 12, 47 (1992).

6. M.P. Bernstein et al. Astrophys. J. 454, 327 (1995).

7. M.P. Bernstein et al. Science 283, 1135 (1999).

8. M.P. Bernstein et al. Nature 416, 401 (2002).

9. G.M. Muñoz Caro et al., Nature 416, 403 (2002).

10. M. Nuevo et al. Advances in Space Research 48, 1126 (2011).

11. E.I. Chiang, P. Goldreich, Astrophys.l J. 490, 368 (1997)

12. J.S. Carr, J.R. Najita, Science 319, 1504 (2008).

13. F.J. Ciesla, Astrophys. J. 723, 514 (2010).

14. F.J. Ciesla, Astrophys. J. 740, 9 (2011).

15. S. Charnoz, et al., Astrophys. J. 737, 33 (2011). 
16. Materials and Methods are described in the Supporting online Material.

17. F.J. Ciesla, J.N. Cuzzi, Icarus 1818, 178 (2006).

18. T. Sano et al., Astrophys. J. 543, 486 (2000).

19. L. Hartmann, et al. Astrophys J. 495, 385 (1998).

20. S.A. Sandford, et al. Science 314, 720 (2006).

21. K. Nakamura-Messenger et al. Science 314, 1439 (2006).

22. K. Lodders, Astrophys. J. 591, 1220 (2003).

23. K. Willacy, Astrophys J. 660, 441 (2007).

24. Z. Zhu, L. Hartmann, C. Gammie, Astrophys. J. 694, 1045 (2009).

25. H.J. Habing, Bulletin of the Astronomical Institutes of the Netherlands 19, 421 (1968).

26. K. Öberg, et al., Astrophys. J. 693, 1209 (2009).

27. T.J. Bethell, E.A. Bergin, Astrophys. J. 739, 78 (2011).

28. F.C. Adams et al., Astrophys. J. 641, 504 (2006).

29. H.B. Throop, Icarus 212, 885 (2011).

30. S.J. Weidenschilling, Icarus 60, 553 (1984).

31. P. Woitke, I. Kamp, W.-F. Thi, Astron Astrophys. 501, 383 (2009).

FJC acknowledges funding from NASA's Origins of Solar Systems program. SAS acknowledges funds from NASA's Origins of Solar Systems Program and the Astrobiology Institute. 


\section{Supplementary Materials}

www.sciencemag.org

Materials and Methods

Figs. S1, S2, S3, S4, S5, S6, S7, S8, S9, S10, S11

References (32-48) 

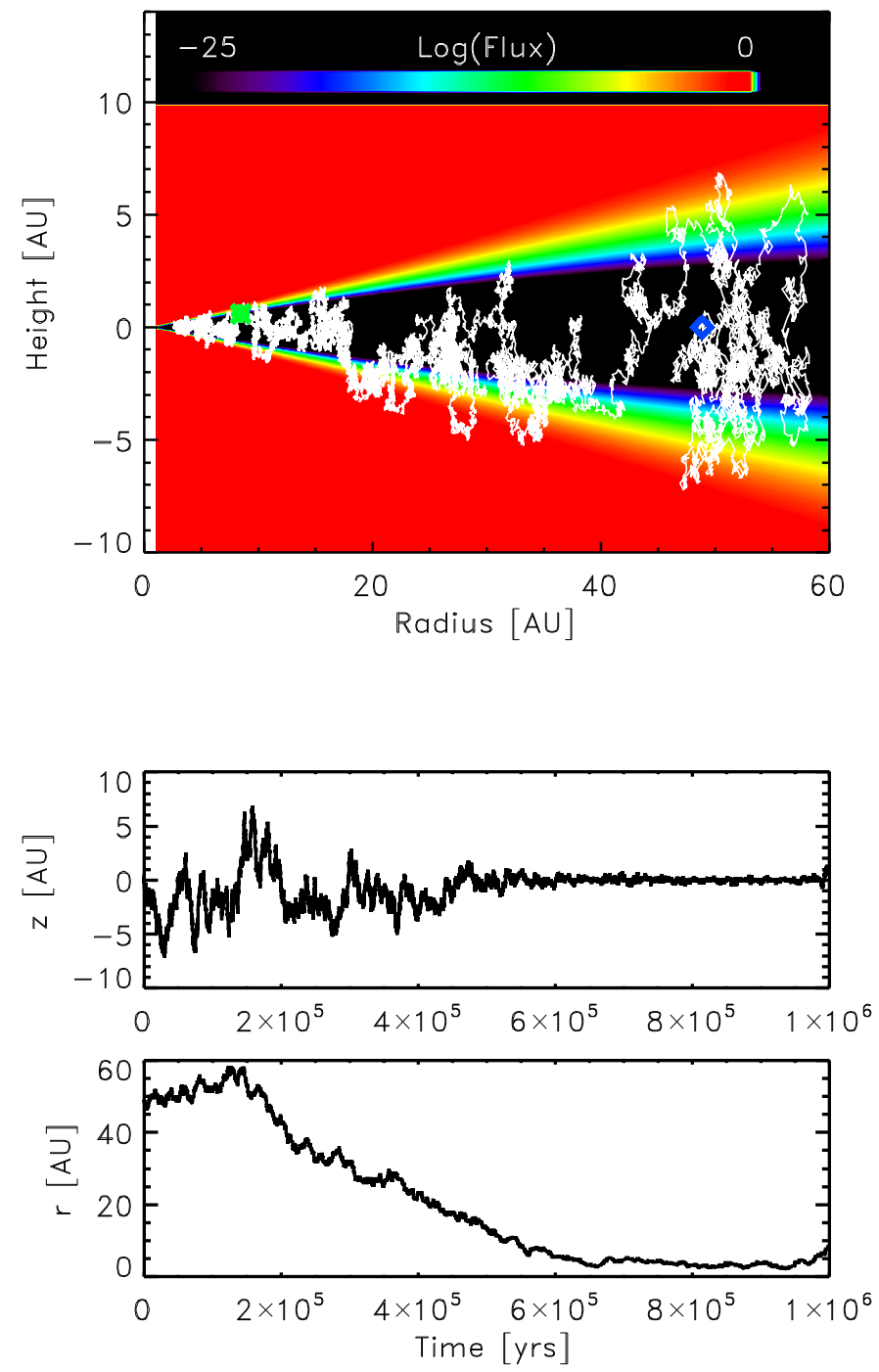

Fig. 1. Top: Trajectory of one of the surviving particles, defined as those that did not migrate inside of $0.1 \mathrm{AU}$ of the young Sun, through the solar nebula superimposed on a map of the UV radiation flux in the disk. The flux is normalized to its value at $z= \pm \propto$ and assumed to propagate normal to the disk midplane. The blue diamond and green asterisk denote the starting and final locations. Bottom: The $r$ and $z$ coordinates of the particle through time. 

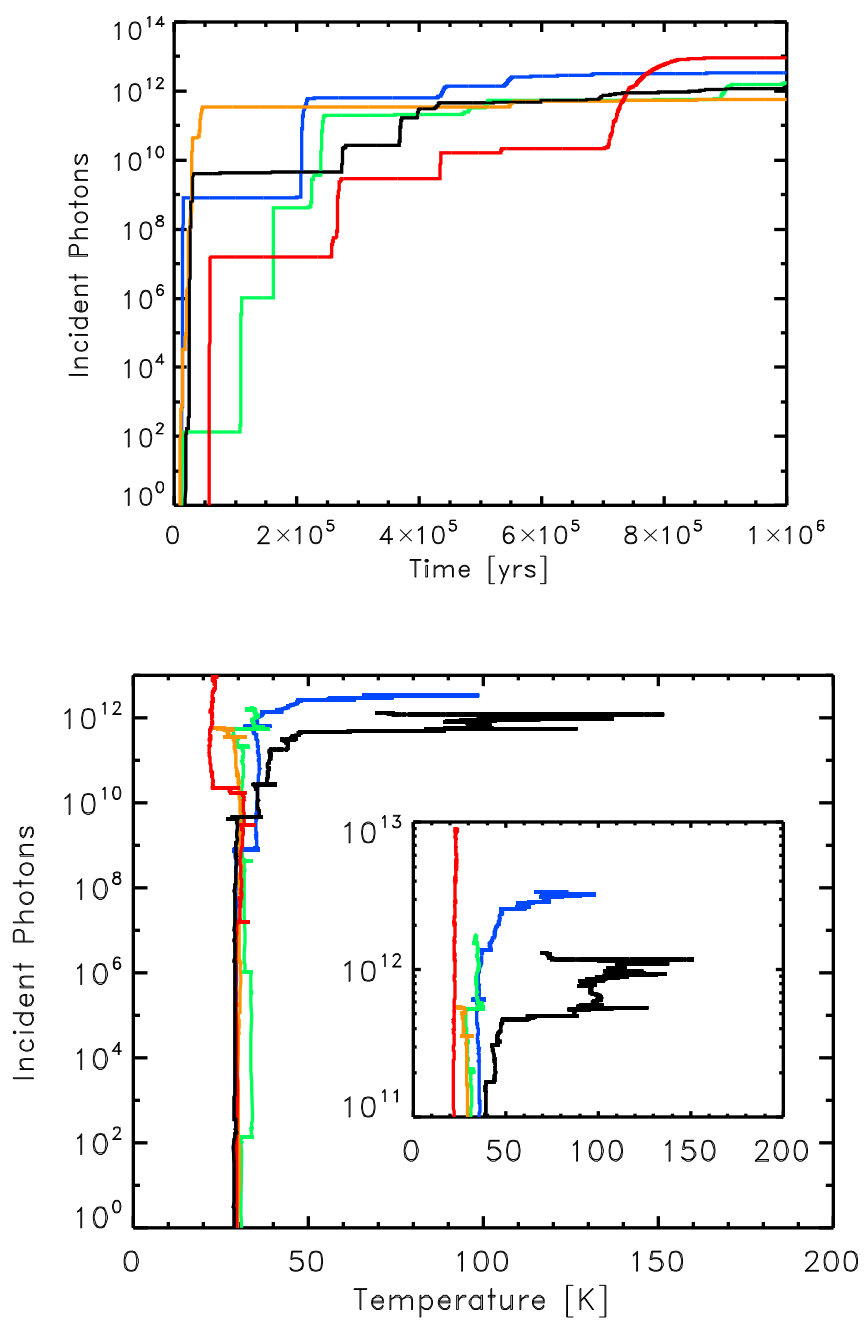

Fig. 2. Top: The cumulative number of photons incident on five grains throughout the $10^{6}$ year lifetime in the disk, assuming an incident flux of $1 G_{0}$. The black line corresponding to the grain whose trajectory is shown in Figure 1. Bottom: Total incident photons seen by the same particles plotted against their temperatures. 


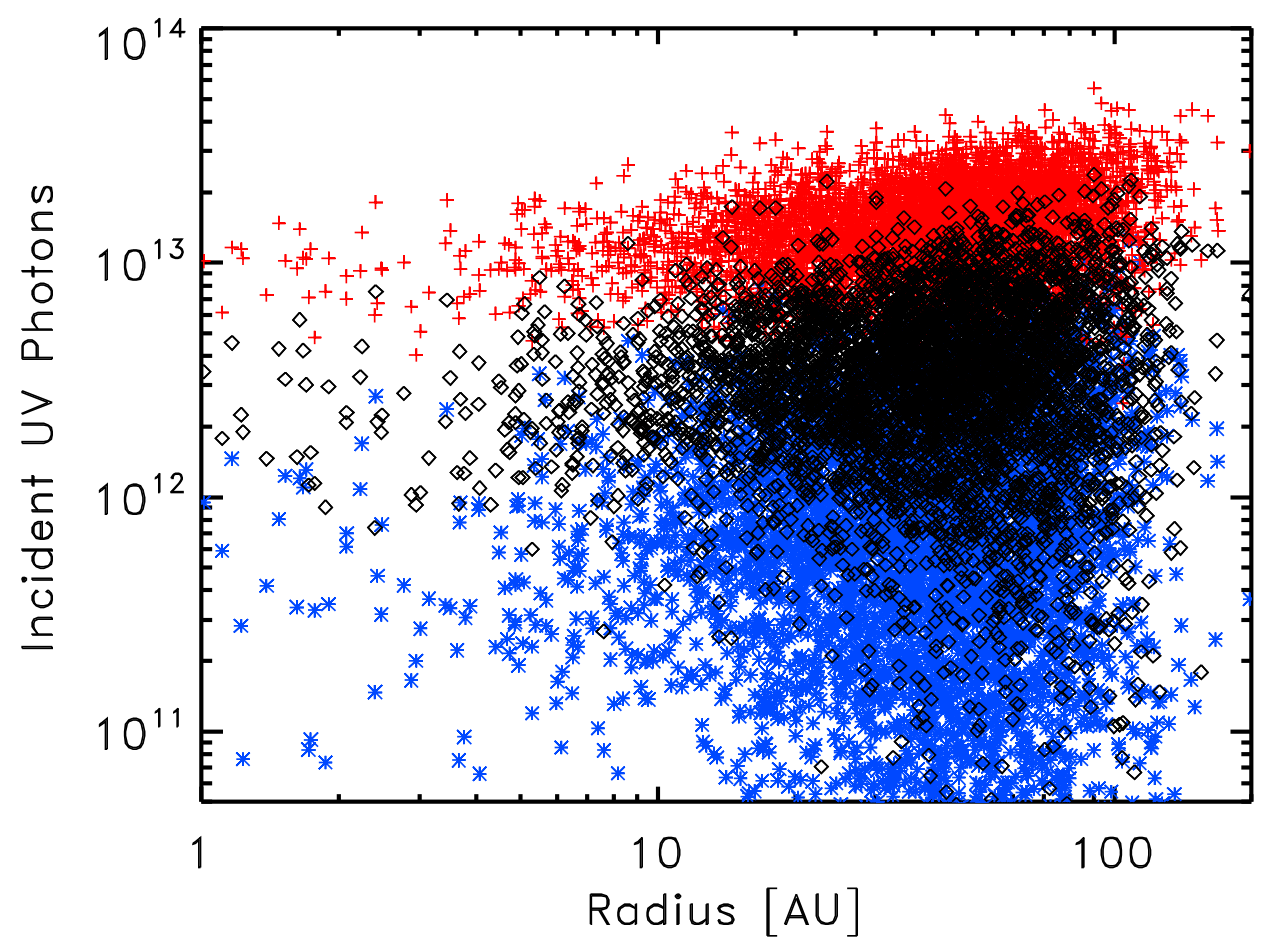

Fig. 3. The total number of incident photons for each of the 4979 surviving grains plotted against their location after $10^{6}$. A typical exposure for our nominal flux (black diamonds) is $\sim 5 \times 10^{12}$ photons. The light red plus signs and blue asterisks represent the same calculations using the lower and higher opacities as described in the text. The variations in average flux due to opacity differences are less than an order of magnitude. 